\title{
Sistema de análisis energético y de temperatura de las ventanas de un aula de clase con y sin aislamiento térmico
}

\section{Energetic and temperature analysis system of classroom windows with and without thermal insulation}

\author{
Carvajal, Rolando ${ }^{*}$, Robles, Jarol ${ }^{1}$, Solís, José ${ }^{1}$, Vargas, Jorge ${ }^{1}$, Marín, Nacarí ${ }^{2}$ \\ ${ }^{1}$ Universidad Tecnológica de Panamá, Centro Regional de Azuero, Licenciatura en Ingeniería Electromecánica \\ ${ }^{2}$ Universidad Tecnológica de Panamá, Centro Regional de Azuero, Facultad de Ingeniería Mecánica
}

\begin{abstract}
Resumen En la mayoría de las aplicaciones de edificios, el conocer los mecanismos de transferencia de calor y sus efectos permite realizar mejoras en los diseños, como por ejemplo, al incrementar el aislamiento en paredes y ventanas se puede lograr un ahorro de energía. En este trabajo se estudia la transferencia de calor en ventanas mediante un sistema experimental denominada 3JR meter, con base en una plataforma Arduino y sensores de temperatura. Se realizaron pruebas en condiciones de clima tropical para la evaluación de las ventanas de un aula de clase del Centro Experimental del Centro Regional de Azuero de la Universidad Tecnológica de Panamá. En este salón se realizaron estudios térmicos para observar el efecto que produce colocar cortinas de poliestireno expandido en un lugar irradiado por el sol y ver cómo esta puede mejorar en cierto modo tanto el confort de las personas como el posible ahorro energético en una unidad de acondicionamiento de aire. También se utilizó un analizador de energía para garantizar un buen funcionamiento del acondicionador de aire y verificar la existencia de variaciones en las tensiones, corrientes o en la frecuencia.
\end{abstract}

Palabras clave Transferencia de calor, medición de temperatura, Arduino, sensor DHT11, analizador de calidad de energía.

\begin{abstract}
In most buildings applications, knowing the mechanisms of heat transfer and its effects allows to make designs improvements, for example, by increasing the insulation in walls and windows can achieve energy saving. In this work we study the heat transfer in windows by an experimental system called 3JR meter, based on an Arduino platform and temperature sensors. Tests for windows insulation evaluation of one classroom of the Experimental Center building of the Regional Center of Azuero, Technological University of Panama, were performed for tropical climate condition. In this room, thermal studies were carried out to observe the effect of expanded polystyrene curtains in a place irradiated by the sun and to see how this can improve in some way both, the people comfort and the possible energy saving in an air conditioning unit. An energy analyzer equipment was also used to ensure good operation of the air conditioner and verify the existence of variations in voltages, currents or frequency.
\end{abstract}

Keywords Heat transfer, temperature measure, Arduino, DHT11 sensor, quality energy analyzer.

* Corresponding Author: rolando.carvajal@utp.ac.pa

\section{Introducción}

En los últimos años el clima de Panamá ha sufrido cambios, debido al cambio climático [1], por lo que es difícil predecir y estudiar su comportamiento. De acuerdo a la Estrategia Nacional de Cambio Climático de Panamá (ENCCP), se requiere un desarrollo de capacidades y de transferencia de tecnologías, "el que busca abordar la escasez de información y tecnologías referentes a los procesos nacionales de adaptación y mitigación, por medio de un robusto fortalecimiento de capacidades y utilización de tecnologías de punta" [2]. En este sentido, se hace necesaria la utilización y/o adaptación de tecnologías para medir diferentes variables relacionadas con el cambio climático. La temperatura en el interior y exterior de un recinto es un ejemplo de ello.

Durante el proceso de diseño, la mayoría no consideran el comportamiento energético de sus proyectos o no cuentan con suficiente tiempo para realizar un balance térmico previo a la etapa de dimensionamiento del sistema de refrigeración. Adicional, el costo de energía para acondicionar un recinto depende de una serie de factores muy diversos, que se relacionan con la localización del proyecto, la exposición al 
sol, la temperatura ambiente, las características de la instalación de la unidad de acondicionamiento de aire y sobre todo el aislamiento térmico (la capacidad de los materiales para oponerse al paso del calor por conducción). La conducción por otra parte es el mecanismo de transferencia de calor por el que el calor se transporta de regiones de alta temperatura a regiones de baja temperatura dentro de un mismo material o entre diferentes cuerpos. Lo antes descrito se encuentra en los edificios, y es por eso que a la hora de realizar proyectos de construcción se deben tomar en cuenta todos estos aspectos, ya que el proyectista requiere métodos sencillos y rápidos para evaluar decisiones de diseño y comparar las ventajas de soluciones alternativas. Al utilizar un buen aislamiento se puede evitar pérdidas de energía, ya que un recinto bien aislado y acondicionado térmicamente garantiza un menor gasto energético durante todo el año. Por esto, es importante sensibilizarnos con el ahorro energético, optimizando el consumo energético.

En este artículo se pretende analizar la transferencia de calor entre el exterior e interior de un salón de clase, irradiado por el sol durante el día, a fin de observar cómo afecta el consumo energético de una unidad de acondicionamiento de aire. El estudio se ha llevado a cabo en la Universidad Tecnológica de Panamá, Centro Regional de Azuero, específicamente en un aula del Centro Experimental(CE-10). Este salón y los que se encuentran a su lado están más expuestos al sol en horas cercanas al medio día, debido a su orientación. En este salón se realizaron estudios térmicos para observar el efecto que produce colocar cortinas en un lugar irradiado por el sol y ver cómo esta puede mejorar en cierto modo tanto el confort de las personas como el posible ahorro energético en una unidad de acondicionamiento de aire. Este trabajo se basa en un trabajo previo realizado en el Centro Experimental [3].

\section{Métodos y Equipos}

\subsection{Métodos}

Para comprobar la influencia de la conductividad térmica del salón utilizado durante el estudio, así como el efecto que causa la presencia o ausencia de cortinas en las ventanas, se diseñó una prueba experimental denominada 3JR meter, con base en una plataforma Arduino con sensor de temperatura DHT11. Durante la prueba se instalaron dos sensores, uno en el interior y el otro en el exterior del salón. En este estudio no se consideró la influencia que tiene el movimiento de personas dentro del aula y su influencia en la transferencia de calor, por lo que durante las pruebas se verificó que en la misma no se tuvieran clases programadas.

Como parte del estudio, se realizaron mediciones del consumo de energía de la unidad de acondicionamiento de aire tipo Split, utilizando un analizador de calidad de energía.
El confort de las personas se evaluó a través de una encuesta, realizada en formularios google.

Las pruebas de medición de temperatura y del consumo energético se hicieron con las luces encendidas, de manera que se tuvieran las mismas condiciones. Las pruebas se llevaron a cabo durante dos días consecutivos, un día sin ningún tipo de aislante y al día siguiente con láminas de poliestireno expandido en las ventanas.

\subsection{Equipos}

El Arduino empleado durante el estudio consiste en una placa electrónica basada en el microcontrolador ATmega328. Este cuenta con 14 entradas/salidas digitales, de las cuales seis se pueden utilizar como salidas PWM (modulación por ancho de pulsos) y otras seis son entradas análogas. La placa incluye todo lo necesario para que el microcontrolador haga su trabajo, basta conectarla a un ordenador con un cable USB o con una fuente de alimentación externa.

Los sensores de temperatura DHT11 poseen un coeficiente de calibración que es guardado como un programa en su memoria otp, la cual es usada por el sensor internamente en su proceso de cálculo en la entrada de la señal. Un simple cable conectado de forma serial hace la integración rápida y fácil con el sensor. Su consumo de energía es muy bajo, siendo una buena opción para sus aplicaciones y es uno de los más usados.

De manera más específica posee un rango de medición en temperatura de 0-50 grados Celsius con un factor de precisión para temperatura de más o menos $2 \%$ con un voltaje de funcionamiento de 3 a $5.5 \mathrm{VDC}$. El uso de este sensor posee las características necesarias para el muestreo, ya que nuestras temperaturas tanto ambientes como interiores, se encuentran en el promedio de rango de mediciones de este sensor y que por su costo y accesibilidad cumple con las especificaciones deseadas. Así mismo, las mediciones de temperatura requieren de un dispositivo que cuente con la capacidad de almacenar datos durante la duración de la prueba. Una vez terminado el muestreo, el usuario debe ser capaz de rescatar los datos para su posterior análisis. Estas funciones se cumplen con los sensores DHT11[4].

El dispositivo 3JR meter permite leer datos de temperatura, con base en un sistema embebido con microcontrolador Arduino y sensores de temperatura y de humedad DHT11. A este dispositivo quisimos llamarlo 3JR meter, ya que está adaptado a nuestra necesidad de medición. Este se programó de manera que se tomaran datos cada cinco minutos en un periodo de ocho horas, para establecer rangos aceptables a la hora de tabular y analizar los datos recolectados. Cabe destacar que los datos se recopilaron en la pantalla mediante monitor serial, lo que hace fácil el traslado de información, ya que en este estudio se realizaron pruebas experimentales de corta duración. 


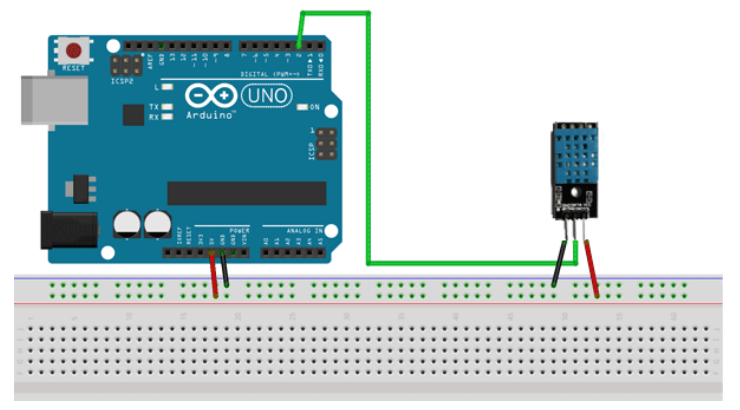

Figura 1. Configuración del 3JR meter.

Para el análisis de energía, se empleó un analizador de calidad de energía AEMC PowerPad Model 3945-B, el cual estaba a nuestra disposición en el campus de la Universidad Tecnológica de Panamá, Sede Azuero. El analizador de calidad de energía empleado en este trabajo es un equipo portátil para análisis y medición de redes de energía eléctrica compacto y resistente a golpes. La toma de datos al acondicionador de aire, en dos ambientes, el primero sin aislante en las ventanas y el segundo con aislante en las ventanas. Cabe destacar que cada medición fue programada para que hiciera captura de datos cada 20 segundos, para un gran manejo de datos y así tener menos porcentajes de error, para que no se diera una alteración a la hora de analizar los resultados. El propósito de esta prueba es ver cuánto sería el ahorro energético en el acondicionador de aire en KWh comparando los dos ambientes antes descritos. Para esto fue necesario localizar el cableado de alimentación del acondicionador y colocar los medidores en sus respectivas posiciones para la posterior medición [5-7].

\subsection{Espacio de prueba}

El espacio utilizado para la prueba experimental con el 3JR meter es un aula de clases dentro del campus de la Universidad Tecnológica de Panamá, sede Azuero (aula CE-10). Cabe destacar que durante el periodo de pruebas el salón no tenía clases programadas, por lo que en el estudio no se tiene el efecto de personas entrando al salón durante las pruebas.

El aula estudiada cuenta con ventanas tipo francesas totalmente transparentes y sin ningún tipo de aislante térmico. Por simple inspección del aula, el sol irradia la misma durante toda la mañana y hasta aproximadamente las 2:30 p. m.

\section{Resultados}

Durante las pruebas, las mediciones en ambos ambientes (ensayo 1 y 2) fueron registradas, obteniéndose 192 datos por ensayo. Se realizaron mediciones en el exterior e interior del salón en dos ambientes diferentes. La temperatura registrada en el exterior del aula de $33^{\circ} \mathrm{C}$. En el caso de la prueba que se realizó sin aislamiento, la temperatura interior fue de $27^{\circ} \mathrm{C}$, mientras que la prueba que se realizó con aislamiento, la temperatura fue de $24^{\circ} \mathrm{C}$.
La tercera y cuarta prueba consistieron en un análisis de calidad de energía, en la que se tomaron medidas de la cantidad de energía consumida con y sin aislante térmico.

Durante estos ensayos, se hizo una captura total de 1440 lecturas, lo que representa 180 datos por cada hora. Estos datos se obtuvieron en KWh. Para la prueba sin aislante se registró un consumo entre 1.6 y $2.4 \mathrm{KWh}$, mientras que para la prueba que se realizó con aislante, el consumo osciló entre 1.4 y 1.9 $\mathrm{KWh}$. Cabe destacar que todas las pruebas se realizaron durante 8 horas de clase del turno diurno y parte del vespertino.

\section{Análisis de mediciones}

Para este análisis se tomó en cuenta la temperatura del ambiente y del interior del salón. Para comprobar y estudiar más a fondo la transferencia de calor que ocurre, debido al cambio de temperatura, se graficaron los datos obtenidos, tanto en el exterior e interior del salón con aislamiento y sin aislamiento (ver figura 4).

Las pruebas con y sin aislamiento dieron como resultado, para el exterior, temperaturas con poca variación. Después de las diez de la mañana, la temperatura se incrementó tan solo dos grados (entre 32 y $34^{\circ} \mathrm{C}$ ).

Para la parte interior utilizando aislante, se puede observar un comportamiento estacionario. De 12:00 m a 4:00 p. m. la temperatura descendió de $27^{\circ} \mathrm{C}$ a $24^{\circ} \mathrm{C}$. La temperatura en el interior del aula, sin aislamiento se mantiene constante a un valor de $28^{\circ} \mathrm{C}$, y cerca de las tres de la tarde, la temperatura desciende a $25^{\circ} \mathrm{C}$ hasta las cuatro de la tarde, hora en que se finalizaron las pruebas.

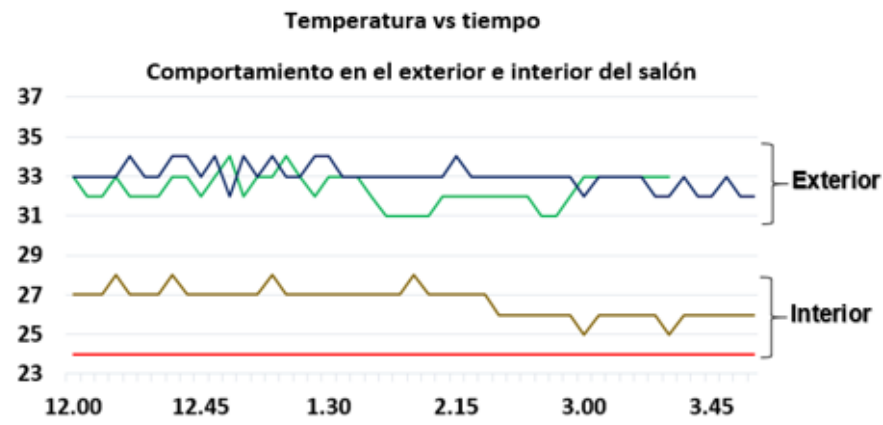

Figura 4. Gráfica de comparación los dos ambientes estudiados con los datos recolectados con el $3 \mathrm{JR}$ meter.

Según los datos recopilados con el 3JR meter se puede observar cuales son los puntos críticos (horas) de temperaturas en el periodo de estudio, así como el comportamiento del aislamiento en las ventanas.

Las pruebas utilizando el analizador de energía se hicieron con la finalidad de observar el consumo de energía a lo largo de ocho horas. Se puede decir que el acondicionador de aire necesita trabajar más de lo necesario para mantener el confort. Los datos arrojan que el consumo mayor se presentó entre las 9:30 y 10:30 de la mañana y de 1:30 a 2:30 de la tarde. 
En la figura 5 se observa la diferencia entre los dos ambientes y cómo influye el uso de aislante en las ventanas, en el aspecto de consumo energético, lo cual a la larga puede representar un ahorro de dinero significante.

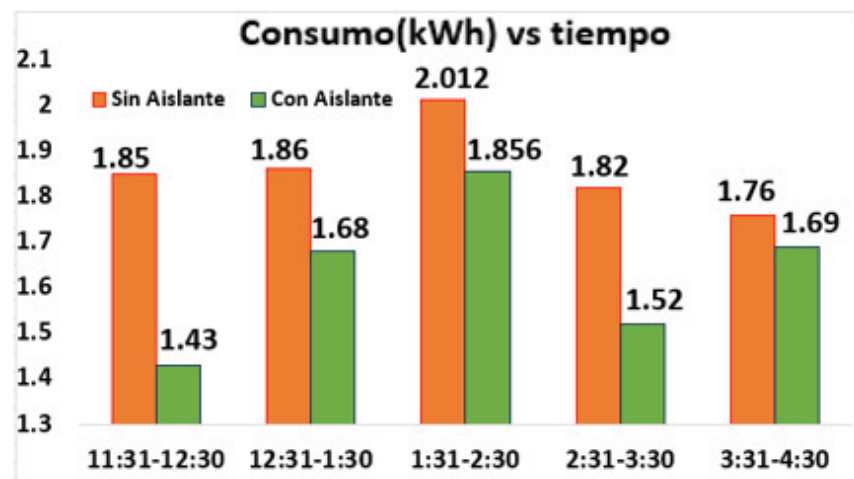

Figura 5. Gráfica de comparación los dos ambientes estudiados con los datos recolectados con el analizador de calidad de energía.

La obtención de estos datos nos dio a entender que sin un aislante el salón está consumiendo más energía, que un salón con aislamiento [8-10].

\section{Confort de las personas}

Se realizó una encuesta a 100 personas dentro de la universidad, en la que se realizaron tres preguntas referentes al confort que se espera tener dentro de un aula de clases.

La primera está referida a la opinión que tiene el participante acerca de tener o no cortinas. Los participantes dieron sus respuestas y se obtuvo un $87 \%$ de personas que prefieren poner cortinas contra un $13 \%$ que prefieren tener el salón expuesto al sol sin cortinas.

Para la segunda pregunta, se tomó como referencia la radiación del sol, y su afectación o problemas que causa a la hora de dar clases. En otras palabras, si suponemos que todo el día está soleado, las radiaciones traen consigo problemas debido a la temperatura del ambiente (generalmente en Panamá está a $31^{\circ} \mathrm{C}$ tratándose de un valor promedio y asumiendo un horario de entre las 11 y 2 de la tarde). Para esta pregunta, un $58 \%$ de personas indicó que les molesta, $13 \%$ no le afecta y el $29 \%$ restante tal vez le moleste.

Por último, en la tercera pregunta se consultó sobre el tipo de iluminación que se prefiere de las opciones dadas la encuesta (en este caso natural o con lámparas), $62 \%$ prefiere las lámparas contra un 38\% que prefieren la luz natural del sol.

\section{Conclusiones}

En las pruebas se consideró la influencia del aislamiento de ventanas tanto en la variación de temperatura interior de un recinto, como en el consumo energético.
Con el aislamiento colocado en las ventanas se logra mantener una estabilidad térmica dentro del entorno estudiado, la que se encuentra tres grados Celsius por debajo respecto a la prueba sin aislante.

A través del análisis de Transferencia de Calor se puede obtener la energía que absorbe el salón a través de la pared y ventanas en forma de calor.

Tras los resultados obtenidos por las encuestas de confort, podemos alegar que los encuestados prefieren un espacio con unas condiciones controladas.

Se deben adoptar iniciativas que incentiven el cambio de las prácticas de consumo, enfocado en el desarrollo de una cultura de ahorro e informar permanentemente sobre los resultados de las acciones de ahorro energético.

Finalmente, con el estudio energético ejecutado se extraen los datos que nos muestran un ahorro de $2.05 \mathrm{KWh}$ que representan $\mathrm{B} /$. 0.38 por día y alrededor de $\mathrm{B} / .7 .70$ por mes, lo que significa un ahorro de B/. 77.04 al año, para el salón estudiado. Se puede asumir que hay cinco salones con las mismas características que el salón de prueba, por lo que se puede estimar un ahorro aproximado de B/. 385.20 al año.

\section{TRABAJO A FUTURO}

Entre las variables que se podrían poner en práctica para continuar con el presente proyecto serían la medición de la humedad en el aula de clase, obtención de una mayor cantidad de datos de temperatura y datos de energía $(\mathrm{KWh})$ para varios días y en diferentes estaciones del año (estación seca y lluviosa), así como el estudio con otros tipos de materiales.

\section{AGRADECIMIENTO}

Gracias al Ing. Roberto Cigarruista por la tutoría brindada para el uso del analizador de calidad de energía y por describirnos ciertos parámetros sobre el ahorro energético.

\section{REFERENCIAS}

[1] Gobierno de la República de Panamá (2014, septiembre 23). [Online].

Disponible: https://www.presidencia.gob.pa/Noticias/Panamapresenta-estrategia-contra-Cambio-Climatico-ante-Cumbresobre-el-Clima-en-Naciones-Unidas-(Última consulta: 15 de junio de 2018).

[2] MI AMBIENTE. (2015, junio 12). Estrategia Nacional de Cambio Climático de Panamá (ENCCP).

Disponible:

http://www.miambiente.gob.pa/images/stories/documentos_CC/ Esp_Info_V.1_ENCCP_15.12.2015.pdf. (Última consulta: 12 de junio de 2018).

[3] A. García, "Diseño de sistema multidimensional de captura y análisis de datos muestreados basado en sistemas embebidos de bajo costo y código abierto." Trabajo de graduación, Universidad Tecnológica de Panamá, 2016. 
[4] A. García, \& K. Navarro (2013, febrero 14). DHT11: Sensor de humedad/temperatura para Arduino. [Online] Disponible: http://panamahitek.com/dht11-sensor-de-humedadtemperaturapara-arduino/.

[5] A. R. Peña, V. A. Gómez, \& C. Hernández, "Diseño y construcción de un prototipo de medición de los indicadores de calidad del servicio de energía eléctrica (DES y FES) para usuario residencial”, Revista Lasallista de Investigación, vol. 7, pp. 101-112, julio-diciembre 2010.

[6] F. Cárdenas \& D. Marcillo, "Auditoria energética eléctrica del campus sur de la Universidad Politécnica Salesiana sede Quito", tesis de pregrado, Universidad Politécnica Salesiana, Quito, Ecuador, 2012.

[7] F. Bozinovic, M. Rosenmann, \& C. Veloso, "Gasto de energía”, Revista Chilena de Historia Natural, Vol.1, pp. 61, 8186. junio 1988.
[8] M. A. Hortal. El ahorro energético: estudios de viabilidad económica. Ediciones Díaz de Santos. 2004.

[9] A. L. León, Muñoz, \& P. Bustamante, "Monitorización de variables medioambientales y energéticas en la construcción de viviendas protegidas: Edificio Cros-Pirotecnia en Sevilla", Consejo Superior de Investigaciones Científicas Informes de la Construcción, Vol. 62, No 519, 2010.

[10] E. Hall., "Medidas de ahorro energético adoptadas en la UTP", Revistas Académicas UTP, Vol. 1, septiembre 2009. Medidas de ahorro energético adoptadas en la UTP”. Universidad Tecnológica de Panamá. 